\title{
Diffusion-weighted imaging and diffusion kurtosis imaging for early evaluation of the response to docetaxel in rat epithelial ovarian cancer
}

\author{
Su-juan Yuan ${ }^{1}$, Tian-kui Qiao ${ }^{1}$ and Jin-Wei Qiang ${ }^{2 *}$
}

\begin{abstract}
Background: To investigate diffusion-weighted magnetic imaging (DWI) and diffusion kurtosis magnetic imaging (DKI) for the early detection of the response to docetaxel (DTX) chemotherapy in rat epithelial ovarian cancer (EOC).

Methods: 7,12-Dimethylbenz[A]anthracene was applied to induce orthotopic EOC in Sprague-Dawley rats. Rats with EOC were treated with DTX on day 0 (treatment group) or were left untreated (control group). DWI and DKI were performed on days $0,3,7,14$ and 21 after treatment. On day 21, the tumors were categorized into the sensitive and insensitive groups according to the size change. The cutoff values of the DWI and DKI parameters for the early response were determined. The experiment was repeated, and the treatment group was divided into the sensitive and insensitive groups according to the initially obtained cutoff values. The DWI and DKI parameters were correlated with tumor size, proliferation, apoptosis and tumor necrosis.

Results: In the sensitive vs. insensitive or control group, significant differences were found in the $\Delta \%$ of the DWI and DKI parameters (ADC, D and $K$ ) from day 3 and in tumor size from day 14 . Early on day 7 , the $\triangle \%$ of $K$ had an AUC of 1 and sensitivity and specificity values of $100 \%$ and $100 \%$, respectively, to detect the response to DTX using a cutoff value of $19.03 \%$ reduction in K. From day 7 , significant differences were found in the $\triangle \%$ of Ki-67 and CA125 in the sensitive vs. control group and from day 14 in the sensitive vs. insensitive group. From day 14 , there were significant differences in the $\Delta \%$ of $\mathrm{BCl}-2$, apoptosis and tumor necrosis in the sensitive vs. control or insensitive group. The $\Delta \%$ values of $A D C$ and $D$ were negatively correlated with the $\triangle \%$ values of tumor size, Ki-67, CA125 and BCl-2 and were positively correlated with the $\Delta \%$ values of apoptosis and tumor necrosis. The $\Delta \%$ of $\mathrm{K}$ was positively correlated with the $\Delta \%$ values of tumor size, Ki-67, CA125 and $\mathrm{BCl}-2$ and was negatively correlated with the $\Delta \%$ values of apoptosis and tumor necrosis.
\end{abstract}

Conclusions: DWI and DKI parameters, especially K, are superior for imaging tumor size for the early detection of the response to DTX chemotherapy in induced rat EOC.

Keywords: Epithelial ovarian cancer, Diffusion-weighted imaging, Diffusion kurtosis imaging, Ki-67, Cancer antigen 125, Docetaxel, Apoptosis, Tumor necrosis

\footnotetext{
*Correspondence: dr.jinweiqiang@163.com

2 Department of Radiology, Jinshan Hospital, Shanghai Medical College, University, 1508 Longhang Road, Shanghai 201508, People's Republic

of China

Full list of author information is available at the end of the article
} 


\section{Background}

Ovarian cancer is one of the most common malignant tumors in female genital organs, and the mortality rate ranks first in gynecological tumors. Epithelial ovarian cancer (EOC) is the most common histological type, accounting for approximately $90 \%$ of malignant ovarian tumors. Despite the development of cytoreductive surgery and chemotherapy,the overall 5-year survival rate of EOC patients remains approximately 40\% [1]. Therefore, developing new therapeutic approaches is imperative. Since the 1990s, docetaxel plus platinum has become the first-line chemotherapeutic regimen of EOC [2]. Although proven effective for EOC, DTX benefits only some patients because of resistance or insensitivity. For non-responders, the traditional response evaluation criteria in solid tumors (RECIST) based on changes in tumor size as well as the marker levels cannot reflect the microstructural changes in the tumor after chemotherapy sufficiently early. Therefore, developing a reliable method to detect a response as early as possible will be significant to avoid noneffective chemotherapy and wasted time.

Magnetic resonance (MR) diffusion-weighted imaging (DWI) offers monitoring prior to morphological changes. Chemotherapy drugs contribute to kill tumor cells, subsequently decreasing the cell density and restricted diffusion motions of water molecules. The study of colorectal cancer by Delli et al. demonstrated that the increased apparent diffusion coefficient (ADC) value of a tumor after chemotherapy was negatively correlated with tumor cell proliferation and was positively correlated with apoptosis [3]. In addition, many studies have demonstrated that DWI is an important biomarker in the prediction and early evaluation of the efficacy of treatment for EOC [4-7]. Diffusion kurtosis imaging (DKI) is an advanced technique based on a non-Gaussian model and can reflect water diffusion deviation from Gaussian behavior [Jensen et al.]. Compared with standard DWI, it can more accurately describe the complicated water diffusivity in biological tissue and provide more information about tissue heterogeneity and cellularity with parameters of D (corrected ADC) and K (diffusion kurtosis) [8]. DKI has mainly been used for the differential diagnosis of benign and malignant tumors [9, 10]. Until now, to the best of our knowledge, DKI has not been applied for the early evaluation and prediction of the chemotherapy response of EOC, except in a few studies concerning glioblastoma and nasopharyngeal carcinoma [11-13]. Therefore, the objective of our study was to investigate whether the ADC value of DWI, and $K$ and D values of DKI can be used to assess the early response to DTX chemotherapy in induced rat EOC by correlating with the tumor size, Ki-67, Bcl-2, apoptosis and tumor necrosis.

\section{Methods}

\section{Tumor model}

All animal experimental procedures were approved by Institutional Animal Care committee of Jinshan Hospital of Fudan University and were performed according to the Guide for the Care and Use of Laboratory Animals of the National Science and Technology Committee of China. Two hundred female Sprague-Dawley (SD) rats (8 weeks old; Shanghai Laboratory Animal Research Center, Shanghai, China) underwent surgery to establish orthotopic EOCs. The surgical procedures and protocol of the induction of EOCs were the same as those in our previous study [14]. After rat was anesthetized, the right ovary was surgically exposed, packed with a piece of absorbable cloth $(0.5 \mathrm{~cm} \times 0.5 \mathrm{~cm})$ coating $2 \mathrm{mg}$ carcinogen 7,12-Dimethylbenz[A] anthracene (DMBA). Twenty rats died in the process of surgery and tumorigenesis. In the remaining 180 rats, 170 rats developed ovarian malignancies. Among them, 138 rats with tumors were randomly selected into our experiment and 117 EOCs were designed into the analysis of our study after excluding 21 non-EOCs.

\section{Experimental design}

The study consisted of training and validation parts (Fig. 1). In the training part (a), 180 days after operation, 24 rats were randomly assigned to treatment $(\mathrm{n}=16)$ and control $(\mathrm{n}=8)$ groups. In the treatment rats, $12 \mathrm{mg} / \mathrm{kg}$ of DTX was administered via the caudal vein [15]. All rats underwent standard DWI and DKI scanning before (day 0 ) and on the 3rd, 7th, 14th, and 21st days after DTX therapy. On day 21 after DTX therapy, rats with EOCs were divided into the sensitive group (tumor with decreased or unchanged size) and insensitive group (tumor with increased size) by referencing the RECIST guidelines and a previous study [16, 17]. DWI and DKI parameters and tumor sizes at different time points were analyzed retrospectively, and Youden's index, the cutoff value that represents predictive factors to assess an early therapy response, was obtained using logistic regression analysis and receiver operating characteristic (ROC) curve analysis. In the validation part (b), the experiment was repeated, and rats received the same DTX therapy and MRI scanning as those in part A. The treatment group was divided into the sensitive and insensitive groups at an optimal time point according to the obtained Youden's index. Several rats in every group at different time points and in the control group on day 0 , respectively, were killed for histopathological and immunohistochemical (IHC) analyses. In both parts, at different time points, only rats with EOCs demonstrated by histopathology were included in the study. In part B, the 

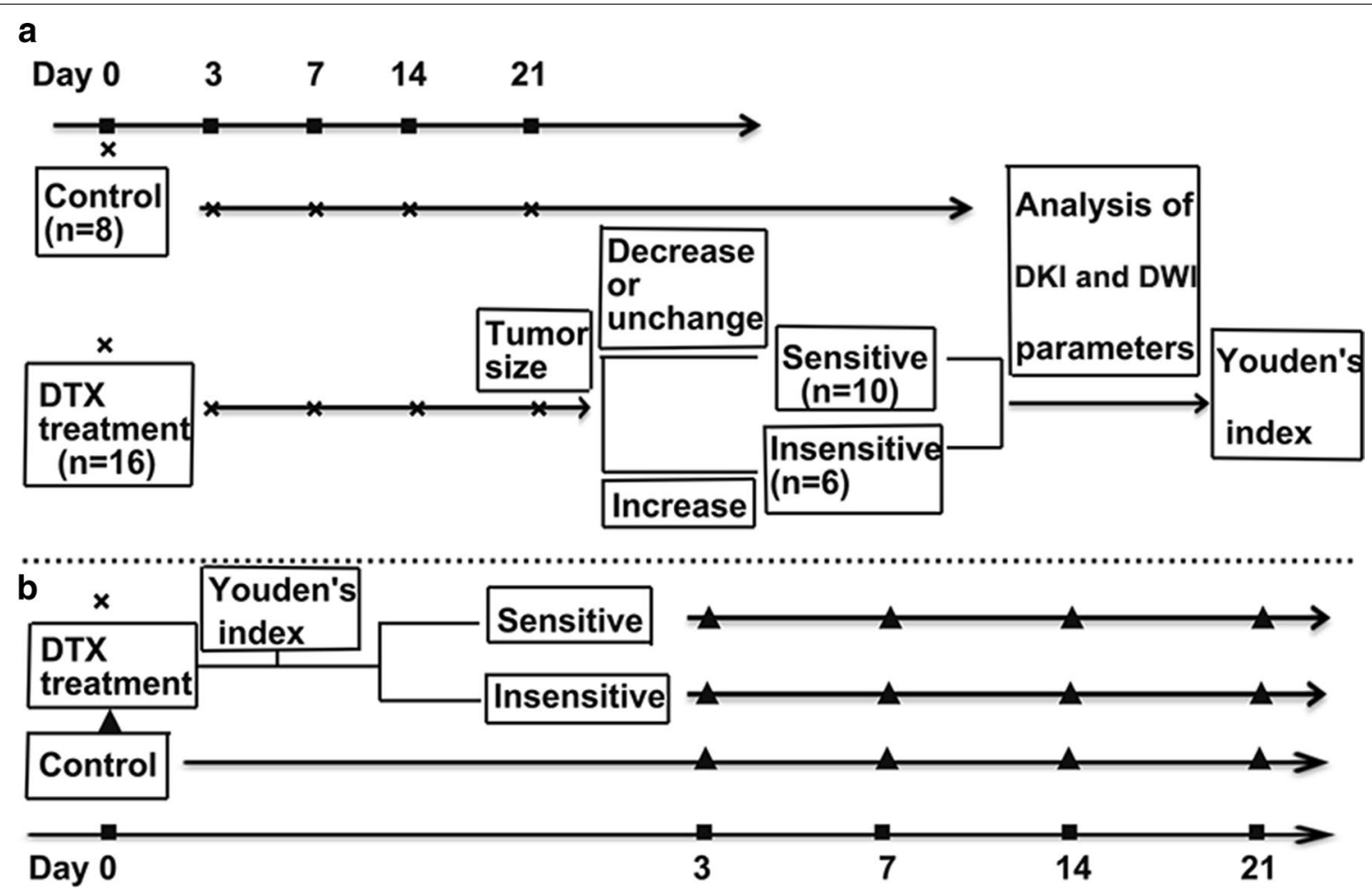

Fig. 1 Flow chart of the study design showing grouping, treatment and imaging time points. a DWI and DKI were performed for all rats on day 0 after which they were randomly assigned to the treatment and control groups. The rats in the treatment group received $12 \mathrm{mg} / \mathrm{kg}$ of DTX. Before (day 0) and 3, 7,14, 21 days after DTX therapy, all rats underwent MRI scanning. Twenty-one days after DTX therapy, the tumors were categorized into the sensitive group and the insensitive group. Youden's index was obtained using retrospective logistic regression analysis of the DWI and DKI parameters at different time points. b Another 93 rats received the same DTX, DWI and DKI as those in $\mathbf{a}$. According to the above cutoff value at the optimal time point, the treatment rats were categorized into the sensitive or insensitive groups. Several rats in the control group on day 0 and in every group at different time points were killed, and the tumors were excised for histology "black up-pointing triangle" indicated DWI, DKI and histology). $\Delta \%$ DWI/DKI parameter $=\left(\right.$ DWI/DKI parameter after - DWI/DKI parameter $\left._{\text {day } 0}\right) / D W I / D K I$ parameter day $0_{0} \times 100 \% . \Delta \%$ tumor size $=($ tumor size $_{\text {after }}-$ tumor size $\left._{\text {day } 0}\right) /$ tumor size day $0 \times 100 \% . \Delta \% \mathrm{Ki}-67 / \mathrm{BCl}-2 /$ apoptosis rate/necrosis rate $=\left(\mathrm{Ki}-67 / \mathrm{Bcl}-2 /\right.$ apoptosis rate/necrosis rate ${ }_{\text {after }}-\mathrm{Ki}-67 /$ $\mathrm{BCl}-2 /$ apoptosis rate/necrosis rate day $_{0}$ )/Ki-67/BCl-2/apoptosis rate/necrosis rate day $0_{0} \times 100 \%$. (Note: The tumor size indicated the longest diameter of the tumor; $\mathrm{Ki}-67_{\text {day } 0}, \mathrm{BCl}-2_{\text {day } 0}$ apoptosis rate day $0_{0}$ and necrosis day 0 represented the average expression of corresponding biomarkers in the control group on day 0 )

Table 1 Number of rats with EOC in different groups and at different time points

\begin{tabular}{lllllc}
\hline Groups & D0 & D3 & D7 & D14 & D21 \\
\hline Treatment group & 48 & & & & \\
Sensitive group & $30(0)$ & $30(0)$ & $30(10)$ & $20(9)$ & $11(11)$ \\
Insensitive group & $18(0)$ & $18(0)$ & $18(7)$ & $11(56)$ & $5(5)$ \\
Control group & $45(9)$ & $36(9)$ & $27(10)$ & $17(8)$ & $9(9)$ \\
\hline
\end{tabular}

The number of rats sacrificed for histopathology is in parentheses

number of rats with EOCs in different groups and at different time points are listed in Table 1.

\section{MRI scanning}

Rats underwent MRI scanning under anesthesia using a $3.0 \mathrm{~T}$ scanner (Verio; Siemens Healthcare, Erlangen, Germany) with a rat coil. The following sequences were obtained: axial spin echo (SE) T1-weighted imaging (T1WI) (time of repetition/time of echo [TR/ $\mathrm{TE}]=7.29 / 2.28 \mathrm{~ms}$ ); axial, sagittal and coronal turbo SE T2W1 with fat saturation $[\mathrm{TR} / \mathrm{TE}=2500 / 93 \mathrm{~ms}]$; and turbo SE T2W1 (TR/TE $=8000 / 98 \mathrm{~ms})$. DWI and DKI were performed using a single-shot echo planar imaging (EPI) sequence. The imaging parameters were: TR/ TE, 3300/71 ms; section thickness, $2 \mathrm{~mm}$; field of view (FOV), $120 \times 64.9 \mathrm{~mm}^{2}$; matrix, $148 \times 148 ; 4 \mathrm{~b}$ factors of DKI: 0, 700, 1400, 1500, $2100 \mathrm{~s} / \mathrm{mm}^{2}$; and acquisition time, $315 \mathrm{~s} .2 \mathrm{~b}$ factors of DWI 0 and $1000 \mathrm{~s} / \mathrm{mm}^{2}$.

\section{DWI and DKI processing}

The DKI and DWI images were independently evaluated by two radiologists with 10 years of experience in pelvic MRI and who were blinded to the histopathological information. The non-Gaussian DKI model was 


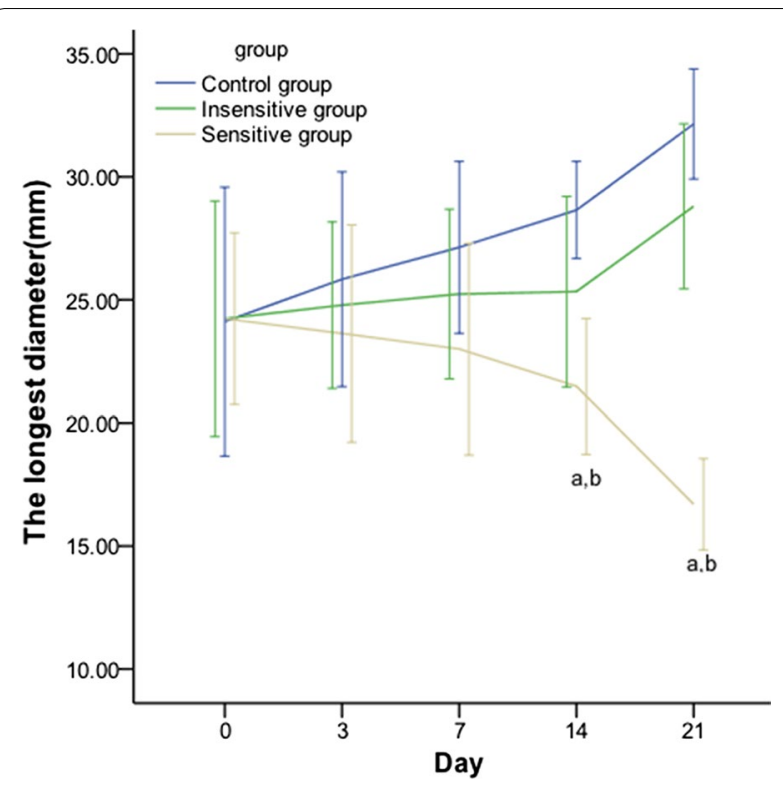

Fig. 2 Line graph showing the effect of DTX on the tumor size in rat EOCs. From day 14 after DTX administration, the tumor size of the sensitive group was significantly decreased compared with that of the insensitive and control counterparts. The tumor size indicates the longest diameter. $p<0.05{ }^{\mathrm{a}}$ : sensitive vs. control; ${ }^{\mathrm{b}}$ : sensitive vs. insensitive

mathematically expressed by the following nonlinear equation: $S_{b}=S_{0} \cdot \exp \left(-b \cdot D+1 / 6 b^{2} \cdot D^{2} \cdot K\right)$, in which $S_{b}$ is the DWI signal intensity at a particular b value, and $S_{0}$ is the signal intensity when the b value is $0 \mathrm{~s} / \mathrm{mm}^{2}$. D represents the corrected ADC of the non-Gaussian distribution behavior, and $\mathrm{K}$ is the apparent diffusional kurtosis, a dimensionless parameter that reflects the deviation of diffusion from Gaussian behavior. D and $\mathrm{K}$ were calculated using in-house software (MatLab; MathWorks, Natick, MA, USA). ADC maps were generated from the standard DWI with b values of 0 and $1000 \mathrm{~s} / \mathrm{mm}^{2}$.

\section{Histopathological and IHC analysis}

In the validation part (b), after completing MRI scanning at every time point, the rat ovary was removed and fixed for HE staining to evaluate histopathology and tumor necrosis, and for IHC staining to investigate the expression of Ki-67 and Bcl-2, as previously described [14]. Tumor necrosis was semi-quantitatively analyzed using Image-Pro Plus 6.0 imaging software [18]. Tumor necrosis rate $=$ necrosis area/field area $\times 100 \%$. The tumor necrosis, Ki-67 and $\mathrm{Bcl}-2$ statuses in three randomly selected high-power fields $(\mathrm{HPF} \times 200)$ were obtained and averaged.

\section{ELISA}

At the target time point, the evaluation of cancer antigen 125 (CA125) in murine serum was applied with ELISA as previously described [14].

\section{Flow cytometry analysis}

EOC tissues were made into single cell suspension that were incubated with FITC-conjugated Annexin-V and propidium iodide for $15 \mathrm{~min}$ at room temperature. Immediately, the cells were analyzed using a Cytomics ${ }^{\mathrm{TM}}$ FC500 flow cytometer (Beckman Coulter, Inc., Fullerton, CA, USA). The data were analyzed using Summit version 5.2 software (Beckman Coulter, Inc.). Apoptosis rate $=$ number of pieces of apoptosis cells/total number of tumor cells.

\section{Statistical analysis}

The data were analyzed using SPSS 22.0 (Chicago, IL, USA) and are presented as the mean \pm standard deviation. One-way analysis of variance (ANOVA) was used for data analysis between multiple groups, and the differences between every two group were analyzed by the least significant difference test (LSD-t). Spearman's correlation was used to analyze the correlation between the change rates of DWI and DKI parameters and change rates of the tumor size, necrosis, apoptosis, $\mathrm{Ki}-67$ and

Table 2 Change rates in the tumor size at different time points (\%)

\begin{tabular}{lcccc}
\hline Group & Day 3 & Day 7 & Day 14 & Day 21 \\
\hline Sensitive & $-2.17 \pm 5.35$ & $-6.27 \pm 3.80^{\mathrm{a}}$ & $-16.69 \pm 2.96^{\mathrm{a}, \mathrm{b}}$ & $-30.46 \pm 3.05^{\mathrm{a}, \mathrm{b}}$ \\
Insensitive & $4.09 \pm 6.69$ & $4.85 \pm 3.72$ & $10.57 \pm 5.61$ & $20.58 \pm 4.24$ \\
Control & $8.53 \pm 4.33$ & $14.37 \pm 4.543$ & $21.11 \pm 4.67$ & $37.49 \pm 9.64$ \\
\hline
\end{tabular}

Tumor size: longest diameter

$p<0.05$

a Sensitive vs. control; ${ }^{\text {b }}$ Sensitive vs. insensitive 

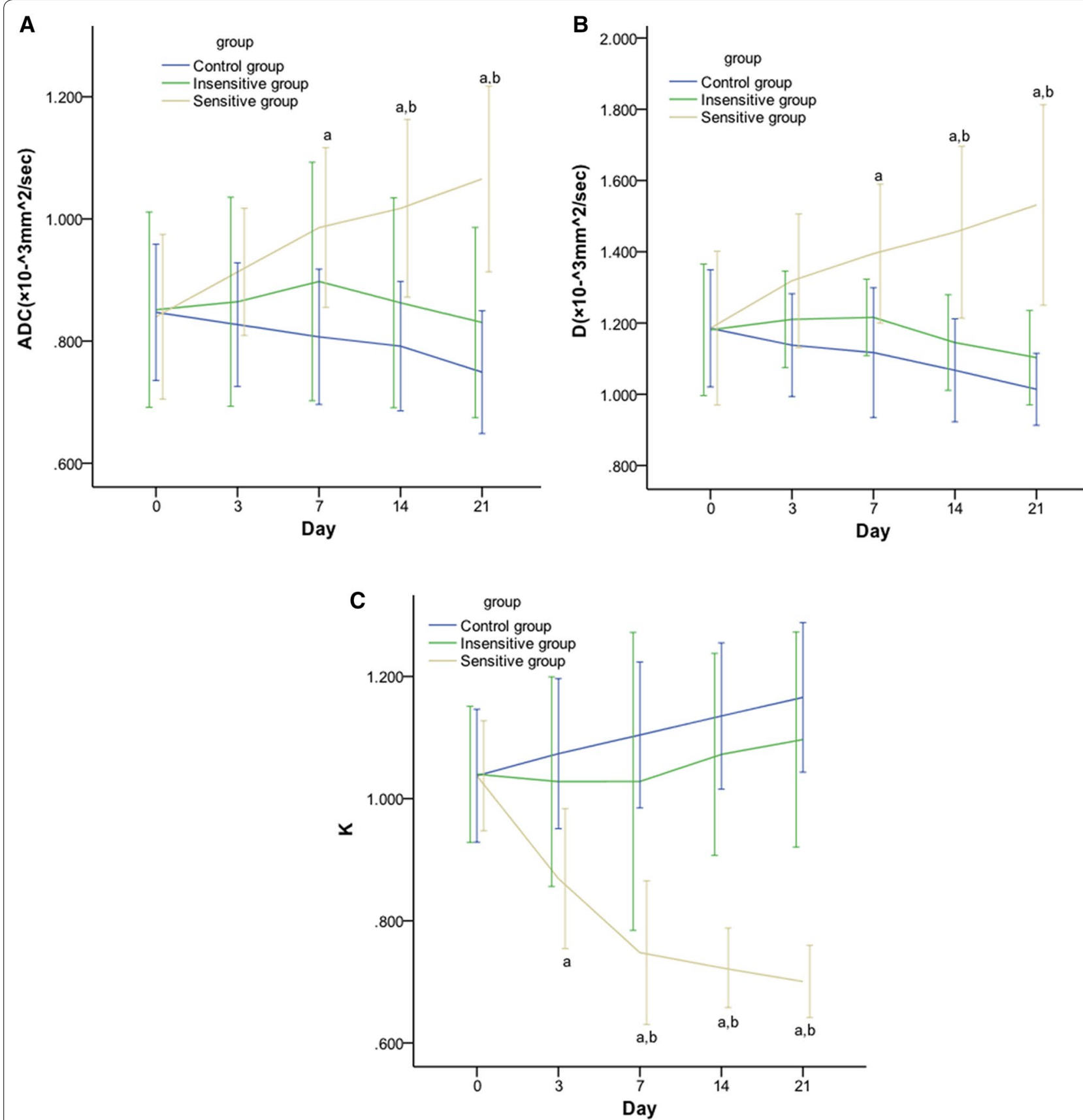

Fig. 3 Line graphs showing the effect of DTX on DWI and DKI parameters in rat EOCs. From day 7 after DTX administration, ADC (A) and D (B) values of the sensitive group were significantly decreased compared with those of the insensitive and control counterparts from day $14 . \mathrm{K}(\mathbf{C})$ value of the sensitive group is significantly decreased compared to its insensitive counterpart from day 3 and to control group from day 14 . $p<0.05$, a. sensitive vs. control; ${ }^{\text {b }}$ sensitive vs. insensitive

Bcl-2. The results were interpreted according to the degree of association as strong $(1 \geq r \geq 0.75)$, moderate $(0.75>r \geq 0.50)$, low $(0.50>r \geq 0.25)$, or not relevant $(\mathrm{r}<0.25)$ after taking significant correlation $(p<0.01$ or $p<0.05)$ values into consideration [19-21]. A $p<0.05$ was considered statistically significant. 
Table 3 Change rates of DWI and DKI parameters at different time points (\%)

\begin{tabular}{llccc}
\hline Time & Group & \multicolumn{1}{c}{$\Delta \% \mathrm{ADC}$} & \multicolumn{1}{c}{$\Delta \% \mathrm{D}$} & \multicolumn{1}{c}{$\Delta \% \mathrm{~K}$} \\
\hline Day 3 & Sensitive & $20.55 \pm 2.40^{\mathrm{a}, \mathrm{b}}$ & $22.44 \pm 5.65^{\mathrm{a}, \mathrm{b}}-16.28 \pm 2.99^{\mathrm{a}, \mathrm{b}}$ \\
& Insensitive & $1.39 \pm 1.54$ & $3.16 \pm 2.89$ & $-1.61 \pm 2.24$ \\
& Control & $-2.22 \pm 1.85$ & $-3.81 \pm 1.63$ & $3.42 \pm 0.95$ \\
Day 7 & Sensitive & $17.94 \pm 2.61^{\mathrm{a}, \mathrm{b}}$ & $18.55 \pm 3.48^{\mathrm{a}, \mathrm{b}}$ & $-28.22 \pm 1.95^{\mathrm{a}, \mathrm{b}}$ \\
& Insensitive & $5.09 \pm 3.28$ & $4.01 \pm 3.87$ & $-2.14 \pm 4.90$ \\
& Control & $-4.57 \pm 3.31$ & $-5.81 \pm 2.94$ & $6.42 \pm 1.07$ \\
Day 14 & Sensitive & $21.67 \pm 3.14^{\mathrm{a}, \mathrm{b}}$ & $23.39 \pm 5.36^{\mathrm{a}, \mathrm{b}}$ & $-30.32 \pm 0.57^{\mathrm{a}, \mathrm{b}}$ \\
& Insensitive & $1.50 \pm 4.22$ & $-2.34 \pm 3.62$ & $2.80 \pm 2.50$ \\
& Control & $-6.37 \pm 2.94$ & $-9.78 \pm 2.47$ & $9.48 \pm 1.84$ \\
Day 21 & Sensitive & $27.41 \pm 3.56^{\mathrm{a}, \mathrm{b}}$ & $29.33 \pm 3.70^{\mathrm{a}, \mathrm{b}}-32.43 \pm 1.13^{\mathrm{a}, \mathrm{b}}$ \\
& Insensitive & $-2.18 \pm 3.78$ & $-6.05 \pm 2.87$ & $5.09 \pm 2.97$ \\
& Control & $-11.38 \pm 2.63$ & $-13.75 \pm 3.60$ & $12.42 \pm 2.02$ \\
\hline
\end{tabular}

$\triangle \%$ : change rate

$p<0.05$

a Sensitive vs. control

b Sensitive vs. insensitive
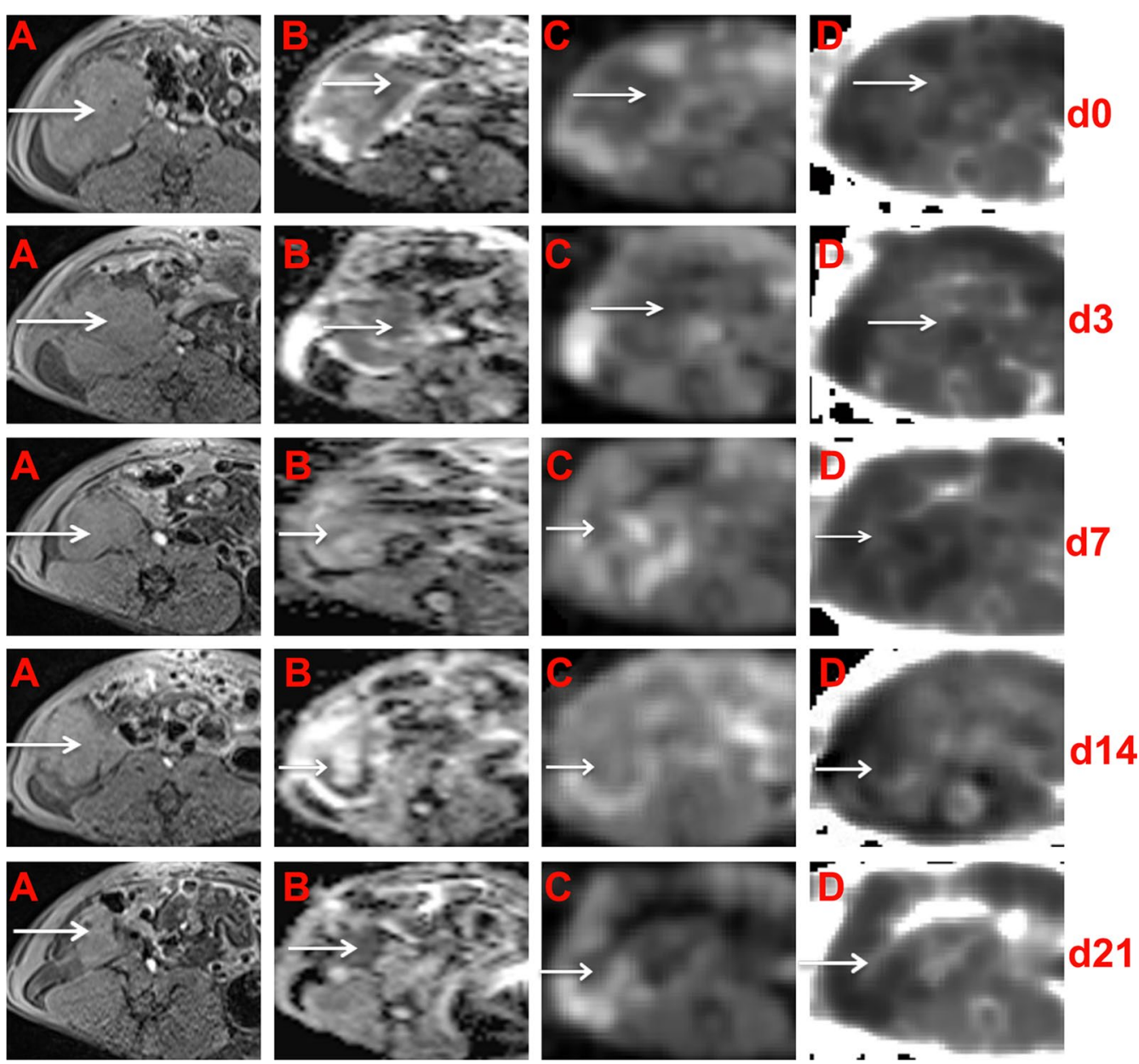

Fig. 4 Conventional, DWI and DKI of EOCs in sensitive rats at different time points. In the right adnexal area, a multilocular cystic-solid EOC (arrow) progressively decreased on TIWI (A). ADC maps were generated from standard DWI (B) and D maps (C) and K (D) maps were generated from DKI. The solid component) showed hypointensity on the ADC maps, hyperintensity on the K maps and hypointensity on the D maps 

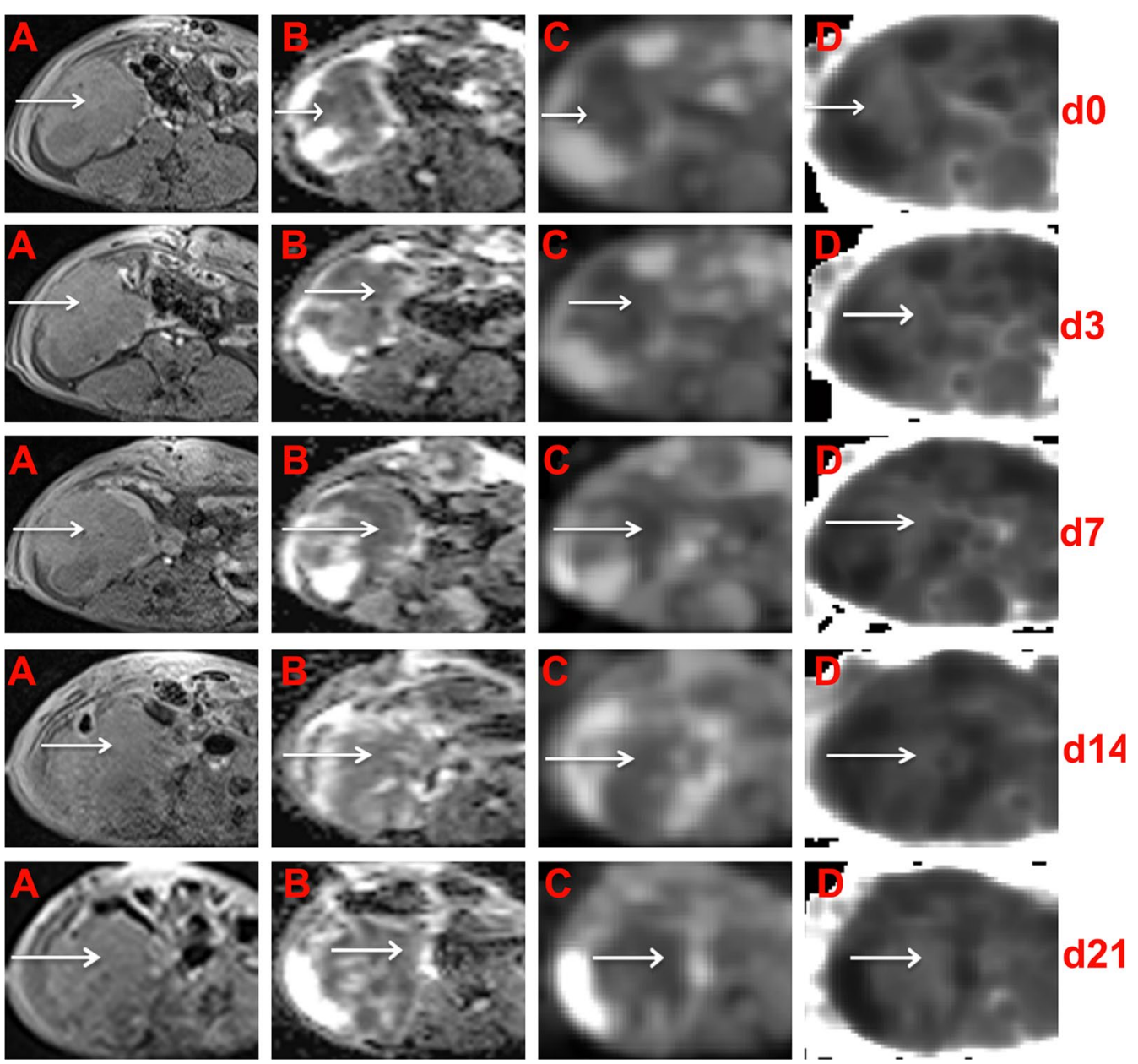

Fig. 5 Conventional, DWI and DKI of EOCs in sensitive rats at different time points. In the right adnexal area, a multilocular cystic-solid EOC (arrow) progressively increased on T1WI (A). ADC maps were generated from standard DWI (B), and D maps (C) and K (D) maps were generated from DKI. The solid component showed hypointensity on the ADC maps, hyperintensity on the K maps and hypointensity on the D maps

\section{Changes in the DWI and DKI parameters after DTX therapy} in rat $\mathrm{EOC}$

On days $0, A D C$ of $\mathrm{DWI}$, and $\mathrm{D}$ and $\mathrm{K}$ of $\mathrm{DKI}$ in rat EOC showed no significant differences among the three groups. On day 3, there was a significant difference in $\mathrm{K}$ in the sensitive vs. control group. On day 7 , significant differences were found in $\mathrm{K}$ in the sensitive vs. control or insensitive group, and in ADC and D in the sensitive vs. control group. On days 14 and 21, significant differences were found in the pairwise comparisons, except for ADC and $\mathrm{D}$ in the insensitive vs. control group (Fig. 3). On days $3,7,14$ and 21, significant differences were found in the pairwise comparisons in the $\Delta \%$ values of all three parameters (ADC, D and K), except for ADC, D and $\mathrm{K}$ in the insensitive vs. control group (Table 3, Figs. 4, 5).

\section{ROC evaluation based on DWI and DKI parameters}

On days 0 and 3 , the ADC, D and $K$ could not evaluate the response to DTX therapy in rat EOC. $\mathrm{K}$ showed its ability to evaluate the efficacy of DTX in EOC, with AUC, sensitivity and specificity values of $0.917,83.3 \%$ and $83.3 \%$, respectively, on day 7 , and values of $1,100 \%$ and $100 \%$, respectively, on day 14 . However, early on day 7 , the change rate of $\mathrm{K}$ showed perfect ability to evaluate the efficacy of DTX in EOCs, with AUC, sensitivity 
A

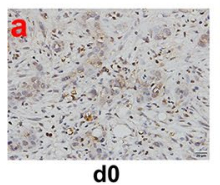

do
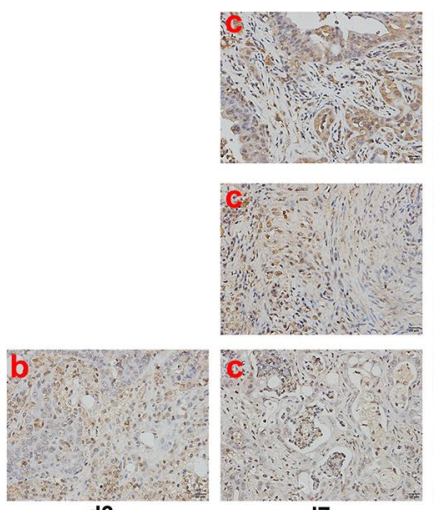

d3

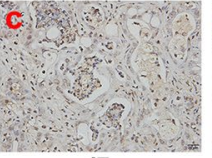

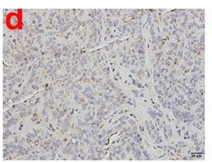
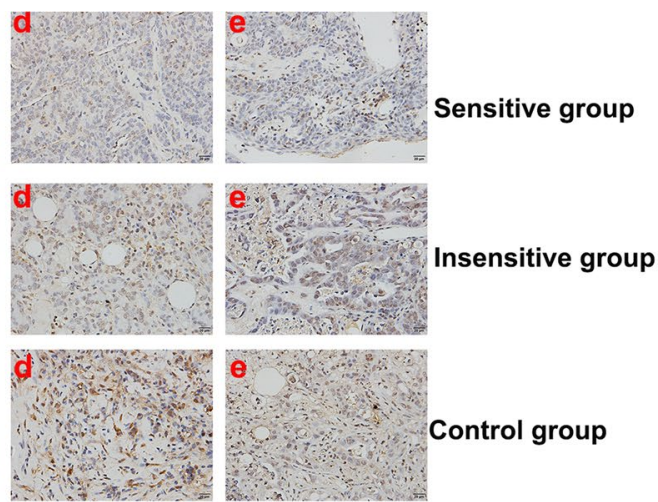

d14

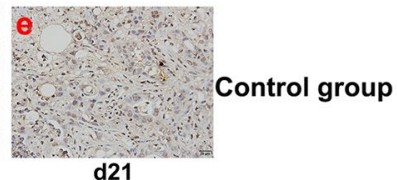

B a
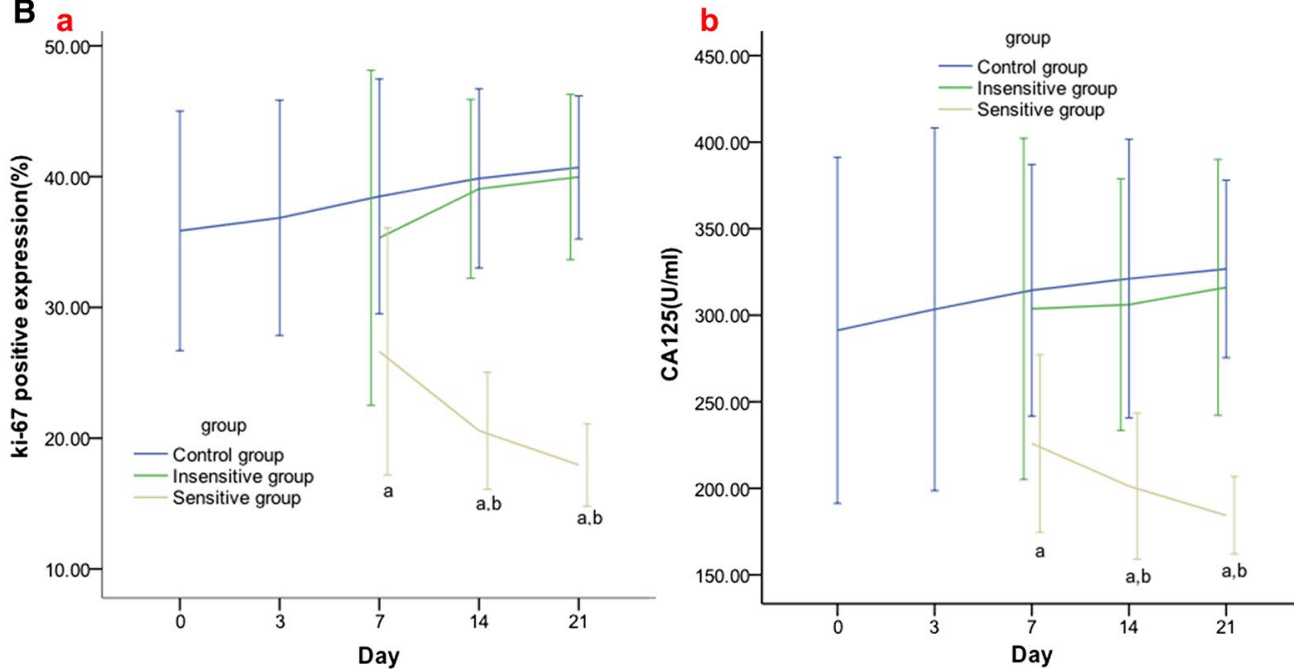

Fig. 6 Effect of DTX on Ki-67 and CA125 in rat EOCs. IHC staining (HRF $\times 200)$ on day 0 (a), day 3 (b), day 7 (c), day 14 (d), and day 21 (e) of Ki-67 (A), which manifest as yellow or brown granules and appear in Ki-67-positive cell nuclei were analyzed. The Ki-67 expression and CA125 level (B) were decreased in the sensitive group but increased in the insensitive or control group. From day 7 , significant differences were found in Ki-67 and CA125 in the sensitive vs. control group and in the sensitive vs. insensitive group from day 14. $p<0.05$, ${ }^{a}$ : sensitive vs. control; b. sensitive vs. insensitive

Table 4 Change rates of $\mathrm{Ki}-67$ expression and the serum CA125 level at different time points (\%)

\begin{tabular}{llcc}
\hline Time & Group & $\boldsymbol{\Delta} \%$ Ki-67 & \multicolumn{1}{c}{$\boldsymbol{\Delta \% \text { CA125 }}$} \\
\hline Day 7 & Sensitive group & $-25.75 \pm 10.26^{\mathrm{a}}$ & $-22.45 \pm 10.26^{\mathrm{a}}$ \\
& Insensitive group & $-1.52 \pm 11.22$ & $4.27 \pm 10.63$ \\
& Control group & $7.32 \pm 9.01$ & $7.94 \pm 8.99$ \\
Day 14 & Sensitive group & $-42.65 \pm 4.86^{\mathrm{a}, \mathrm{b}}$ & $-30.90 \pm 5.64^{\mathrm{a}, \mathrm{b}}$ \\
& Insensitive group & $8.94 \pm 6.00$ & $5.11 \pm 7.84$ \\
& Control group & $11.16 \pm 6.87$ & $10.24 \pm 9.96$ \\
Day 21 & Sensitive group & $-49.93 \pm 3.41^{\mathrm{a}, \mathrm{b}}$ & $-36.71 \pm 2.99^{\mathrm{a}, \mathrm{b}}$ \\
& Insensitive group & $11.47 \pm 5.54$ & $8.51 \pm 7.97$ \\
& Control group & $13.50 \pm 5.49$ & $12.18 \pm 6.34$ \\
\hline
\end{tabular}

\section{$\Delta \%$ : change rate}

$p<0.05$

a Sensitive vs. control

b Sensitive vs. insensitive and specificity values of $1,100 \%$ and $100 \%$, respectively. Youden's index was a 19.03\% reduction in K.

\section{Effective DTX therapy decreases Ki-67 expression and the $\mathrm{CA} 125$ level in rat EOC}

According to the results of validation part (B), on day 7 , the treatment group was divided into the sensitive and insensitive groups, with a $19.03 \%$ reduction in K. Ki-67 expression and the serum CA125 level, and the $\Delta \%$ of Ki-67 expression and serum CA125 level were significantly different between the sensitive and control groups on day 7 and were significantly different between the sensitive vs control or insensitive group on days 14 and 21 (Fig. 6 and Table 4). 


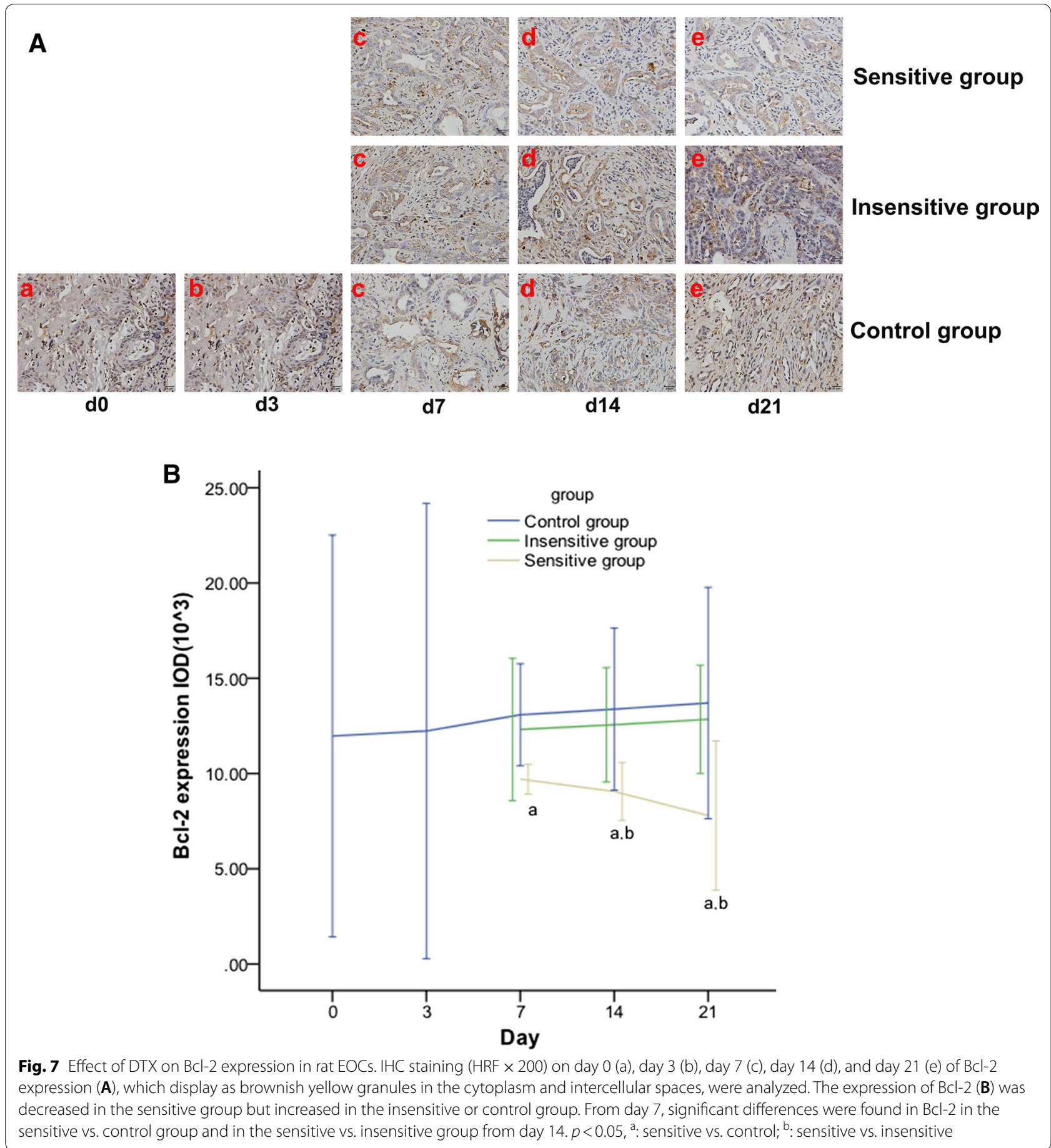

\section{Effective DTX therapy decreases Bcl-2 expression in rat}

\section{EOCs}

Bcl-2 expression and $\Delta \%$ of $\mathrm{Bcl}-2$ expression were significantly different between the sensitive and control groups on day 7, and between the sensitive and control or insensitive groups on days 14 and 21 (Fig. 7 and Table 5). 
Table 5 Change rates of $\mathrm{Bcl}-2$ expression at different time points (\%)

\begin{tabular}{|c|c|c|c|}
\hline Group & Day 7 & Day 14 & Day 21 \\
\hline Sensitive group & $-18.94 \pm 2.53^{a}$ & $-24.34 \pm 4.95^{a, b}$ & $-34.88 \pm 12.73^{a, b}$ \\
\hline Insensitive group & $2.85 \pm 9.81$ & $4.93 \pm 7.88$ & $7.30 \pm 7.47$ \\
\hline Control group & $9.31 \pm 8.06$ & $11.76 \pm 12.80$ & $14.45 \pm 18.27$ \\
\hline
\end{tabular}

$\triangle \%$ : change rate

$p<0.05$

a Sensitive vs. control

b Sensitive vs. insensitive

\section{Effective DTX therapy increases tumor apoptosis and necrosis in rat EOCs}

The apoptosis rate and $\Delta \%$ of the apoptosis rate were significantly different between the sensitive and control groups on day 7 , and between the sensitive and control or insensitive groups on days 14 and 21 (Fig. 8 and Table 6). The tumor necrosis and $\Delta \%$ of necrosis lacked significant differences among the three groups on day 7 and showed significant differences between the sensitive and insensitive or control groups on days 14 and 21 (Fig. 9 and Table 6).

\section{Correlations between the change rates of DWI and DKI parameters and change rates of the tumor size, Ki-67, $\mathrm{Bcl}-2$, apoptosis and tumor necrosis}

The $\Delta \%$ of $\mathrm{K}$ showed a highly positive correlation with the $\Delta \%$ of Ki-67 and CA125, moderately positive correlation with the $\Delta \%$ of tumor size and Bcl-2, and moderately negative correlation with the $\Delta \%$ of apoptosis and tumor necrosis. The $\Delta \%$ values of ADC and D were highly positively correlated with the $\Delta \%$ values of Ki-67 and CA125, moderately positively correlated with the $\Delta \%$ values of tumor size and Bcl-2, and moderately negatively correlated with the $\Delta \%$ values of apoptosis and tumor necrosis (Table 7).

\section{Discussion}

Presently, DWI is the most widely used functional magnetic resonance imaging (fMRI) technique clinically. DKI is the development of DWI with high b values. Although rarely studied in animal models and clinical trials, DKI has shown its superiority in evaluating the efficacy of treatment for malignant tumors compared with DWI [22]. DWI and DKI can detect lesions by probing the random motion of water molecules in tissues and reflect the tumor cell density, cell membrane integrity and heterogeneity. Therefore, DWI and DKI can be applied theoretically to monitor the response to chemotherapy by quantifying the change in tumor microstructures.

Although RECIST is most commonly used to determine the curative effect of the tumor [23], morphology changes in the tumor are often not obvious and occur late. Our results demonstrated that neither the tumor size nor its change rate achieved a perfect result of an AUC of 1 , a sensitivity of $100 \%$, and a specificity of $100 \%$ to monitor the response of EOCs to DTX until day 21.

$\mathrm{Wu}$ et al. found a significantly increased ADC and $\mathrm{D}$, and a decreased $\mathrm{K}$ in patients with cervical nonHodgkin's lymphoma who were sensitive to treatment at 7 days after chemotherapy [24]. Yu J et al. found that increased ADC and D were presented in patients with locally advanced rectal cancer who were sensitive to treatment after neo-adjuvant chemotherapy, and $\mathrm{D}$ was superior to ADC in evaluating treatment sensitivity [25]. However, DKI has not been used yet to evaluate the early response to radiation and chemotherapy. Our results demonstrated a significantly higher change rate of $\mathrm{K}$, and lower change rates of $\mathrm{D}$ and $\mathrm{ADC}$ in the DTX-sensitive group than those in the insensitive and control groups from day 3 to day 21 . However, differences in these parameters could not be found until day 7,14 or 21 . Early on day 7, the change rate of $\mathrm{K}$ had an AUC of 1 , and sensitivity and specificity values of $100 \%$ and $100 \%$, respectively, to detect the response to DTX using a cutoff value of $19.03 \%$ reduction in K. Compared with DWI and DKI parameters (K, D and ADC), the change rates of these parameters could reflect the changes in the tumor microstructures and function earlier, and more sensitively detect the response to chemotherapeutic agents in rat EOC. Additionally, the change rates of $\mathrm{K}, \mathrm{D}$ and $\mathrm{ADC}$ could more effectively and earlier reflect the effective DTX treatment than the change rate of size and would be more reliable biomarkers to 

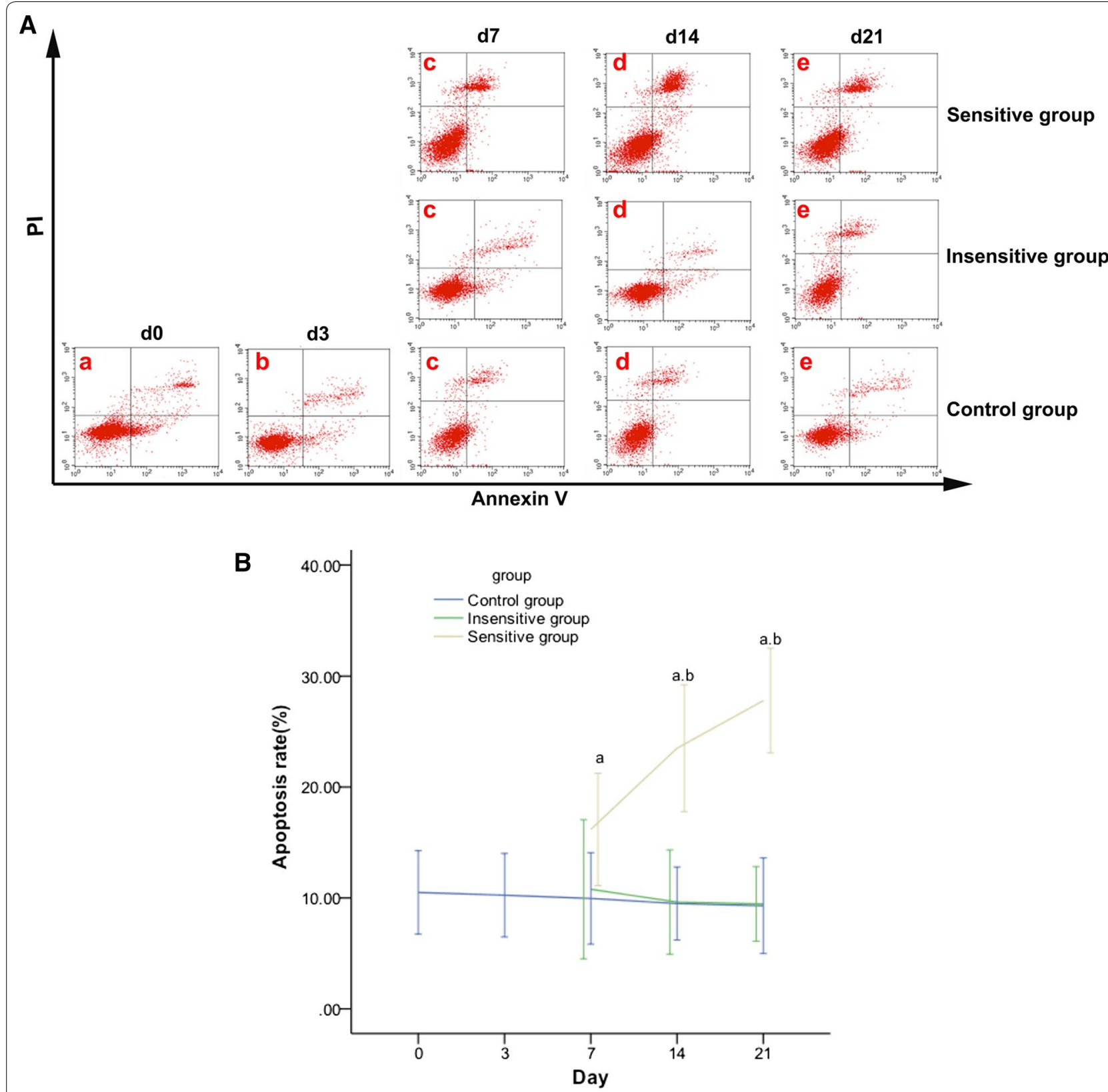

Fig. 8 Effect of DTX on tumor apoptosis in rat EOC. On day 0 (a), day 3 (b), day 7 (c), day 14 (d), day 21 (e), apoptosis (A) was evaluated using Annexin $\mathrm{V}$-fluorescein isothiocyanate/PI staining and flow cytometric analysis. Representative flow cytometry results: bottom right quadrant, cells stained primarily by Annexin V (early apoptotic cells); top right quadrant, cells stained by PI and Annexin V (late apoptotic cells); top left quadrant, cells stained primarily by PI (necrotic cells); bottom left quadrant, cells negative for Annexin V and PI. Apoptosis (B) was increased in the sensitive group but showed no remarkable change in the insensitive or control group. From day 7, significant differences were noted in apoptosis in the sensitive vs. control group and in the sensitive vs. insensitive group from day 14. $p<0.05^{\text {a }}{ }^{\text {: }}$ sensitive vs. control; ${ }^{\text {b: }}$ sensitive vs. insensitive 
Table 6 Change rates of the apoptosis rate and tumor necrosis at different time points (\%)

\begin{tabular}{llcc}
\hline Time & Group & $\boldsymbol{\Delta} \%$ apoptosis rate & $\boldsymbol{\Delta} \%$ necrosis rate \\
\hline Day 7 & Sensitive group & $54.12 \pm 18.76^{\mathrm{a}}$ & $91.36 \pm 26.09$ \\
& Insensitive group & $2.70 \pm 18.76$ & $26.22 \pm 20.22$ \\
& Control group & $-5.17 \pm 14.16$ & $20.27 \pm 16.10$ \\
Day 14 & Sensitive group & $123.91 \pm 21.20^{\mathrm{a}, \mathrm{b}}$ & $172.62 \pm 20.53^{\mathrm{a}, \mathrm{b}}$ \\
& Insensitive group & $-8.28 \pm 14.07$ & $36.64 \pm 13.21$ \\
& Control group & $-9.53 \pm 16.18$ & $27.88 \pm 11.09$ \\
Day 21 & Sensitive group & $164.80 \pm 17.52^{\mathrm{a}, \mathrm{b}}$ & $271.25 \pm 20.21^{\mathrm{a}, \mathrm{b}}$ \\
& Insensitive group & $9.92 \pm 10.08$ & $44.14 \pm 10.16$ \\
& Control group & $-11.43 \pm 19.99$ & $38.26 \pm 10.16$ \\
\hline
\end{tabular}

$\Delta \%$ : change rate

$p<0.05$

a Sensitive vs. control

b Sensitive vs. insensitive

monitor the tumor response to chemotherapy and help make individualized therapy decisions.

ADC is closely correlated with tissue edema, necrosis, apoptosis and fibrosis, which reflect the internal structure and microenvironment of the tumor [26, 27]. Effective treatment can increase the ADC value of the tumor, and the increased ADC is correlated with tumor necrosis and apoptosis [28-30]. Based on the non-Gaussian distribution of water molecules, D is the corrected $\mathrm{ADC}$ and $\mathrm{K}$ is the mean kurtosis that reflects the deviation of diffusion from the Gaussian distribution. $\mathrm{K}$ increases with the complexity of the internal composition of the diseased tissue. A higher $\mathrm{K}$ indicates more complicated tumor microstructures. Our study indicated that effective DTX therapy could increase ADC and D, and decrease $\mathrm{K}$. The explanation could be due to DTX inhibiting cell proliferation, inducing tumor necrosis and apoptosis [31-33], and consequently, increasing the extracellular space, movement of water molecules, ADC and D, and decreasing the complexity of the internal composition of the tumor and $\mathrm{K}[34]$.

$\mathrm{Ki}-67$ is one of the most widely used markers for cell proliferation and malignancy [35]. CA125 is the clinically used biomarker for ovarian cancer and can reflect cell differentiation and progression of ovarian carcinoma, and can reflect the response to chemotherapy [36, 37]. Studies have shown that Ki-67-positive expression and the CA125 level play a significant role in the development, treatment and overall prognosis of EOC [38-40].

Previous studies have suggested that paclitaxel could reduce the Ki-67-positive expression and CA125 level of human ovarian carcinoma xenografts in nude mice [41, 42]. Our study in induced EOCs showed that K could effectively divide the DTX treatment group into the sensitive and insensitive groups on day 7. Ki-67-positive expression and the CA125 level were markedly decreased in the sensitive group compared with those in the insensitive group on day 7 and the control group on day 14 . Furthermore, the change rates of Ki-67 and CA125 were negatively correlated with those of $\mathrm{ADC}$ and $\mathrm{D}$ and were positively correlated with the change rates of $\mathrm{K}$. Our study suggested that ADC, D and K could noninvasively reflect the expression of tumor biomarkers in vivo and treatment-induced changes in the proliferative activity prior to changes in tumor morphology.

Bcl-2 contributes to stimulating cell proliferation and inhibiting apoptosis [43, 44]. A study by $\mathrm{Wu} \mathrm{H}$ et al. showed that ADC was negatively correlated with $\mathrm{Bcl}-2$ in rabbit soft-tissue VX2 carcinoma 5, 10, and 15 days after receiving radiotherapy [45]. Our study indicated that effective DTX could decrease $\mathrm{Bcl}-2$ expression and induce the apoptosis of EOCs. Bcl-2 expression changes were negatively correlated with the changes in ADC and $\mathrm{D}$ and were positively correlated with the change of $\mathrm{K}$. Apoptosis changes were positively correlated with the changes in ADC and D and were negatively correlated with the change in K. Additionally, our study indicated that treatment-induced apoptosis occurred prior to changes in the tumor size. We conjectured that the bursting of cell membranes and apoptosis relieved the movement limitation of water molecules, thus increasing ADC and $\mathrm{D}$ and decreasing $\mathrm{K}$.

We also observed that, 14 days after DTX therapy, tumor necrosis was marked and occurred after changes in the DWI and DKI parameters (D, ADC and K). The change in necrosis was positively correlated with the changes in ADC and D and was negatively correlated with the change in $\mathrm{K}$. Thus, we believe that tumor necrosis increases the extracellular space and movement of water molecules.

Our study had some limitations. First, we used a 3.0 T clinical MR scanner. A 7.0 T or higher field strength MR scanner will improve the image resolution. Second, respiratory gating should be used to improve the accuracy of DKI and DWI parameters. 


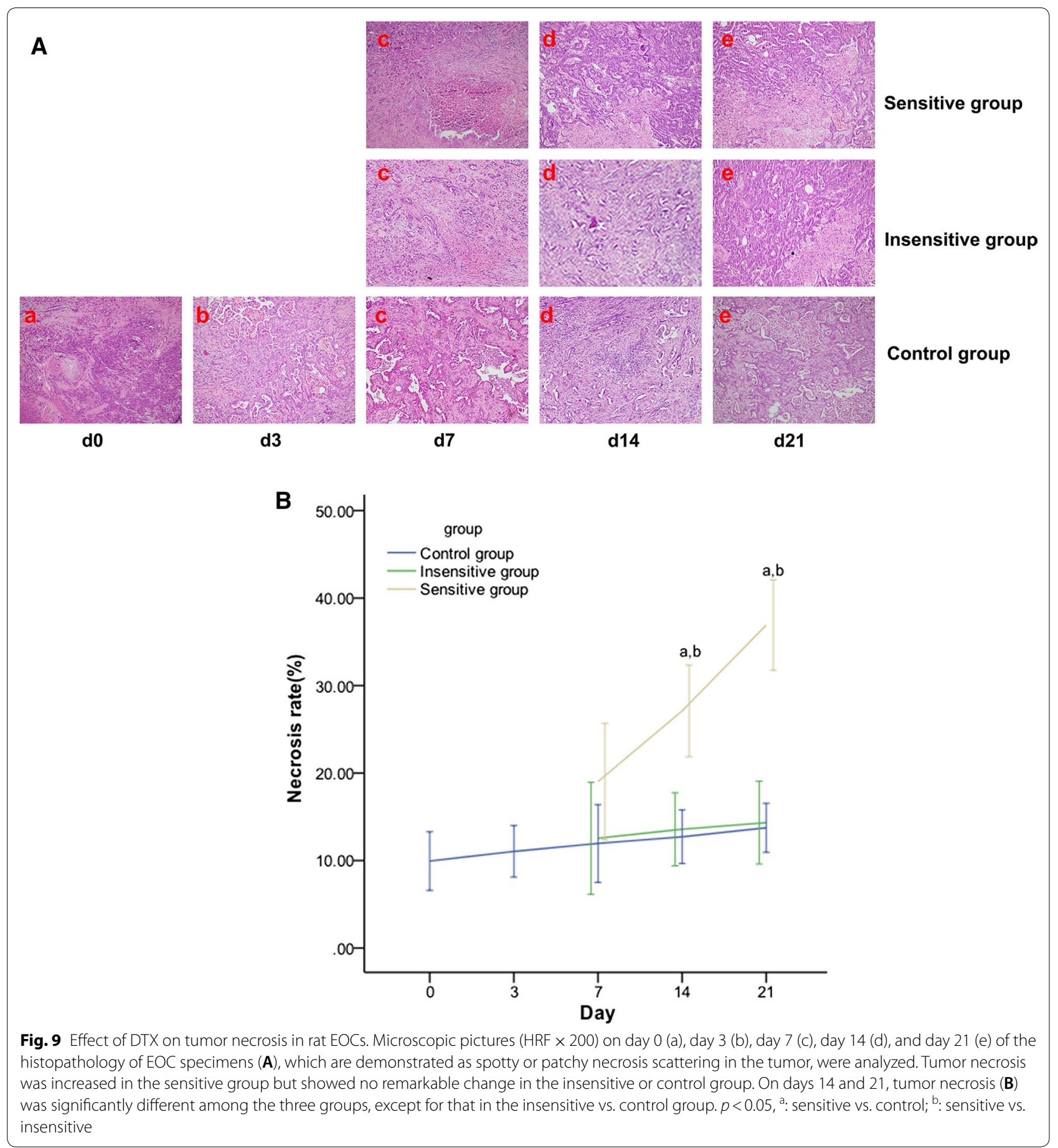

\section{Conclusions}

In conclusion, quantitative parameters of DWI and DKI, especially $\mathrm{K}$, were superior to pathological and molecular biomarkers and imaging tumor size for the early detection and prediction of a response to DTX chemotherapy in EOC. Quantitative parameters of DWI and DKI could contribute to adjusting the treatment regimen for non-responders as soon as possible and improving 
Table 7 Correlation between the $\Delta \%$ of the DWI and DKI parameters and $\Delta \%$ of the tumor size, Ki-67, CA125, Bcl-2, apoptosis and necrosis

\begin{tabular}{|c|c|c|c|c|c|c|}
\hline & \multicolumn{2}{|c|}{$\triangle \% A D C$} & \multicolumn{2}{|l|}{$\Delta \% \mathrm{D}$} & \multicolumn{2}{|l|}{$\Delta \% \mathrm{~K}$} \\
\hline & $r$ & $p$ & $r$ & $p$ & $r$ & $p$ \\
\hline$\Delta \%$ tumor size & -0.546 & $<0.001$ & -0.558 & $<0.001$ & 0.594 & $<0.001$ \\
\hline$\Delta \% \mathrm{Ki}-67$ & -0.788 & $<0.001$ & -0.810 & $<0.001$ & 0.812 & $<0.001$ \\
\hline$\triangle \%$ CA 125 & -0.697 & $<0.001$ & -0.672 & $<0.001$ & 0.751 & $<0.001$ \\
\hline$\Delta \% \mathrm{BCl}-2$ & -0.482 & $<0.001$ & -0.435 & 0.001 & 0.531 & $<0.001$ \\
\hline$\Delta \%$ apoptosis & 0.710 & $<0.001$ & 0.728 & $<0.001$ & -0.705 & $<0.001$ \\
\hline$\Delta \%$ tumor necrosis & 0.718 & $<0.001$ & 0.745 & $<0.001$ & -0.749 & $<0.001$ \\
\hline
\end{tabular}

Tumor size: longest diameter; $\Delta \%$ : change rate

the prognosis. However, it will take some time to apply the thought into large, randomized clinical trials or clinical application.

\section{Abbreviations}

DWl: diffusion-weighted imaging; DKI: diffusion kurtosis imaging; DTX: docetaxel; EOC: epithelial ovarian carcinoma; DMBA: 7,12-dimethylbenz[a] anthracene; RECIST: response evaluation criteria in solid tumors; ADC: apparent diffusion coefficient; H\&E staining: hematoxylin-eosin staining; $I H C$ immunohistochemical; CA125: cancer antigen 125.

\section{Authors' contributions}

Guarantor of the integrity of the entire study, JWQ; study concepts/study design or definition of intellectual content, JWQ and TKQ; data analysis/data acquisition, SJY and SQC; literature research, SJY and JWQ; manuscript editing SJY and JWQ. All authors read and approved the final manuscript.

\section{Author details}

${ }^{1}$ Department of Oncology, Jinshan Hospital, Shanghai Medical College, Fudan University, 1508 Longhang Road, Shanghai 201508, People's Republic of China. 2 Department of Radiology, Jinshan Hospital, Shanghai Medical College, University, 1508 Longhang Road, Shanghai 201508, People's Republic of China.

\section{Acknowledgements}

We would like to thank Dr. Xin Li for his technical assistance in this research.

\section{Competing interests}

The authors declare that they have no competing interests.

\section{Availability of data and materials}

All data generated and analyzed during this study are included in this published article.

\section{Consent for publication}

Not applicable.

\section{Ethics approval and consent to participate}

All protocols were performed in accordance with the ethical claims of the Institutional Animal Care and Ethics Committee of Jinshan Hospital of Fudan University.

\section{Funding}

Grants from: National Natural Science Foundation of China (Grant No. 81471628).

\section{Publisher's Note}

Springer Nature remains neutral with regard to jurisdictional claims in published maps and institutional affiliations.
Received: 18 September 2018 Accepted: 30 November 2018

Published online: 05 December 2018

\section{References}

1. Chen W, Zheng R, Baade PD, Zhang S, Zeng H, Bray F, et al. Cancer statistics in China, 2015. CA Cancer J Clin. 2016:66:115-32.

2. Neijt JP, Engelholm SA, Tuxen MK, Sorensen PG, Hansen M, Sessa C, et al. Exploratory phase III study of paclitaxel and cisplatin versus paclitaxel and carboplatin in advanced ovarian cancer. J Clin Oncol. 2000;18:3084-92.

3. Delli Pizzi A, Cianci R, Genovesi D, Esposito G, Timpani M, Tavoletta A, et al. Performance of diffusion-weighted magnetic resonance imaging at 3.0 T for early assessment of tumor response in locally advanced rectal cancer treated with preoperative chemoradiation therapy. Abdom Radiol 2018;43:2221-30

4. Thoeny HC, Ross BD. Predicting and monitoring cancer treatment response with diffusion-weighted MRI. J Magn Reson Imaging. 2010;32:2-16.

5. Kyriazi S, Collins DJ, Messiou C, Pennert K, Davidson RL, Giles SL, et al. Metastatic ovarian and primary peritoneal cancer: assessing chemotherapy response with diffusion-weighted MR imaging-value of histogram analysis of apparent diffusion coefficients. Radiology. 2011;261:182-92.

6. Sala E, Kataoka MY, Priest AN, Gill AB, McLean MA, Joubert I, et al. Advanced ovarian cancer: multiparametric MR imaging demonstrates response-and metastasis-specific effects. Radiology. 2012;263:149-59.

7. Cebulla J, Huuse EM, Pettersen K, van der Veen A, Kim E, Andersen S, et al. MRI reveals the in vivo cellular and vascular response to BEZ235 in ovarian cancer xenografts with different PI3-kinase pathway activity. Br J Cancer. 2015:112:504-13.

8. Raja R, Sinha N, Saini J, Mahadevan A, Rao KN, Swaminathan A. Assessment of tissue heterogeneity using diffusion tensor and diffusion kurtosis imaging for grading gliomas. Neuroradiology. 2016;58:1217-31.

9. Li HM, Zhao SH, Qiang JW, Zhang GF, Feng F, Ma FH, et al. Diffusion kurtosis imaging for differentiating borderline from malignant epithelial ovarian tumors: a correlation with Ki-67 expression. J Magn Reson Imaging. 2017:46:1499-506

10. LiT, Yu T, Li L, Lu L, Zhuo Y, Lian J, et al. Use of diffusion kurtosis imaging and quantitative dynamic contrast-enhanced MRI for the differentiation of breast tumors. J Magn Reson Imaging. 2018. https://doi.org/10.1002/ jmri.26059.

11. Chakhoyan A, Woodworth DC, Harris RJ, Lai A, Nghiemphu PL, Liau LM, et al. Mono-exponential, diffusion kurtosis and stretched exponential diffusion MR imaging response to chemoradiation in newly diagnosed glioblastoma. J Neurooncol. 2018. https://doi.org/10.1007/s1106 0-018-2910-9.

12. Zheng $D$, Lai G, Chen $Y$, Yue Q, Liu X, Chen $X$, et al. Integrating dynamic contrast-enhanced magnetic resonance imaging and diffusion kurtosis imaging for neoadjuvant chemotherapy assessment of nasopharyngeal carcinoma. J Magn Reson Imaging. 2018. https://doi.org/10.1002/ jmri.26164. 
13. Chen Y, Ren W, Zheng D, Zhong J, Liu X, Yue Q, et al. Diffusion kurtosis imaging predicts neoadjuvant chemotherapy responses within 4 days in advanced nasopharyngeal carcinoma patients. J Magn Reson Imaging. 2015;42:1354-61.

14. Yuan SJ, Qiao TK, Qiang JW, Cai SQ, Li RK. The value of DCE-MRI in assessing histopathological and molecular biological features in induced rat epithelial ovarian carcinomas. J Ovarian Res. 2017;10:65-71.

15. Zhang $P$, Chen L, Zhang Z, Lin L, Li Y. Pharmacokinetics in rats and efficacy in murine ovarian cancer model for solid lipid nanoparticles loading docetaxel. J Nanosci Nanotechnol. 2010;10:7541-4.

16. Rustin GJ, Quinn M, Thigpen T, du Bois A, Pujade-Lauraine E, Jakobsen $A$, et al. Re: New guidelines to evaluate the response to treatment in solid tumors (ovarian cancer). J Natl Cancer Inst. 2004;96:487-8.

17. Zhou LN, Wu N, Liang Y, Gao K, Li XY, Zhang LF. Monitoring response to gefitinib in nude mouse tumor xenografts by (18)F-FDG microPETCT: correlation between (18)F-FDG uptake and pathological response. World J Surg Oncol. 2015;13:111-8.

18. Gan SY, Zhong XY, Xie SM, Li SM, Peng H, Luo F. Expression and significance of tumor drug resistance related proteins and beta-catenin in esophageal squamous cell carcinoma. Chin J Cancer. 2010;29:300-5.

19. Kovač JD, Terzić M, Mirković M, Banko B, Đikić-Rom A, Maksimović R Endometrioid adenocarcinoma of the ovary: MRI findings with emphasis on diffusion-weighted imaging for the differentiation of ovarian tumors. Acta Radiol. 2016;57:758-66.

20. Boge RM, Haugen AS, Nilsen RM, et al. Elderly patients' ( $\geq 65$ years) experiences associated with discharge; Development, validity and reliability of the Discharge Care Experiences Survey. PLoS ONE. 2018;13:e0206904-20.

21. Liu C, Wang K, Chan Q, et al. Intravoxel incoherent motion MR imaging for breast lesions: comparison and correlation with pharmacokinetic evaluation from dynamic contrast-enhanced MR imaging. Eur Radiol. 2016;26:3888-98.

22. Goshima S, Kanematsu M, Noda Y. Diffusion kurtosis imaging to assess response to treatment in hypervascular hepatocellular carcinoma. AJR Am J Roentgenol. 2015;20:W543-9.

23. Eisenhauer EA, Therasse P, Bogaerts J, Schwartz LH, Sargent D, Ford R, et al. New response evaluation criteria in solid tumours: revised RECIST guideline (version 1.1). Eur J Cancer. 2009;45:228-47.

24. Wu R, Suo ST, Wu LM, Yao QY, Gong HX, Xu JR. Assessment of chemotherapy response in non-Hodgkin lymphoma involving the neck utilizing diffusion kurtosis imaging: a preliminary study. Diagn Interv Radiol. 2017;23:245-9.

25. Yu J, Xu Q, Song JC, Li Y, Dai X, Huang DY, et al. The value of diffusion kurtosis magnetic resonance imaging for assessing treatment response of neoadjuvant chemoradiotherapy in locally advanced rectal cancer. Eur Radiol. 2017;27:1848-57.

26. Hamstra DA, Lee KC, Moffat BA, Chenevert TL, Rehemtulla A, Ross BD. Diffusion magnetic resonance imaging: an imaging treatment response biomarker to chemoradiotherapy in a mouse model of squamous cell cancer of the head and neck. Transl Oncol. 2008;1:187-94.

27. Nowosielski M, Recheis W, Goebel G, Güler O, Tinkhauser G, Kostron $\mathrm{H}$, et al. ADC histograms predict response to anti-angiogenic therapy in patients with recurrent high-grade glioma. Neuroradiology. 2011;53:291-302

28. Afaq A, Andreou A, Koh DM. Diffusion-weighted magnetic resonance imaging for tumour response assessment: why, when and how? Cancer Imaging. 2010;10(1A):S179.

29. Zhang Y, Chen JY, Xie CM, Mo YX, Liu XW, Liu Y, et al. Diffusion-weighted magnetic resonance imaging for prediction of response of advanced cervical cancer to chemoradiation. J Comput Assist Tumogr. 2011;35:102-7.

30. Koh DM, Scurr E, Collins D, Kanber B, Norman A, Leach MO, et al. Predicting response of colorectal hepatic metastasis:value of pretreatment apparent diffusion coefficients. Am J Roentgenol. 2007;188:1001-8.

31. Razi Soofiyani S, Mohammad Hoseini A, Mohammadi A, Khaze Shahgoli $\checkmark$, Baradaran B, Hejazi MS. siRNA-mediated silencing of CIP2A enhances Docetaxel activity against PC-3 prostate cancer cells. Adv Pharm Bull. 2017:7:637-43.
32. Tao J, Xu J, Chen F, Xu B, Gao J, Hu Y. Folate acid-Cyclodextrin/Docetaxel induces apoptosis in KB cells via the intrinsic mitochondrial pathway and displays antitumor activity in vivo. Eur J Pharm Sci. 2018;111:540-8.

33. Gharpure KM, Chu KS, Bowerman CJ, Hu Y. Metronomic docetaxel in PRINT nanoparticles and EZH2 silencing have synergistic antitumor effect in ovarian cancer. Mol Cancer Ther. 2014;13:1750-7.

34. Kauppinen RA. Monitoring cytotoxic tumour treatment response by diffusion magnetic resonance imaging and proton spectroscopy. NMR Biomed. 2002;15:6-17.

35. Alves ÂV, Ribeiro DR, Lima SO, Reis FP, Soares AF, Gomes MZ, et al. Expression of Ki-67 and P16 INK4a in chemically-induced perioral squamous cell carcinomas in mice. Rev Col Bras Cir. 2016;43:72-9.

36. Morgado M, Sutton MN, Simmons M, Warren CR, Lu Z, Constantinou PE, et al. Tumor necrosis factor-a and interferon- $\gamma$ stimulate MUC16 (CA125) expression in breast, endometrial and ovarian cancers through NFKB. Oncotarget. 2016;7:14871-84.

37. Bast RC Jr. CA 125 and the detection of recurrent ovarian cancer: a reasonably accurate biomarker for a difficult disease. Cancer. 2010;116:2850-3.

38. Zillhardt M, Park SM, Romero IL, Sawada K, Montag A, Krausz T, et al. Foretinib (GSK1363089), an orally available multikinase inhibitor of c-Met and VEGFR-2, blocks proliferation, induces anoikis, and impairs ovarian cancer metastasis. Clin Cancer Res. 2011;17:4042-51.

39. McCall CM, Shi C, Cornish TC, Klimstra DS, Tang LH, Basturk O, et al. Grading of well-differentiated pancreatic neuroendocrine tumors is improved by the inclusion of both Ki67 proliferative index and mitotic rate. Am J Surg Pathol. 2013;37:1671-7.

40. Liu W, Wang Z, Ma J, Hou Y, Zhao J, Dong B, et al. Elevated serum level of CA125 is a biomarker that can be used to alter prognosis determined by BRCA mutation and family history in ovarian cancer. Genet Test Mol Biomarkers. 2017:21:547-54

41. Hou WJ, Guan JH, Dong Q, Han YH, Zhang R. Dexamethasone inhibits the effect of paclitaxel on human ovarian carcinoma xenografts in nude mice. Eur Rev Med Pharmacol Sci. 2013;17:2902-8.

42. Safra T, Andreopoulou E, Levinson B, Borgato L, Pothuri B, Blank S, et al. Weekly paclitaxel with intermittent imatinib mesylate (Gleevec): tolerance and activity in recurrent epithelial ovarian cancer. Anticancer Res. 2010;30:3243-7.

43. Zhang J, Hu Z, Fang X. MiR-204 inhibits cell growth and induces cell apoptosis though targeting RAB13 in ovarian cancer cells. Int J Clin Exp Pathol. 2016;9:3211-8.

44. Kuwano Y, Nishida K, Kajita K, Satake Y, Akaike Y, Fujita K, et al. Transformer $2 \beta$ and miR-204 regulate apoptosis through competitive binding to $3^{\prime}$ UTR of BCL2 mRNA. Cell Death Differ. 2015;22(5):815-25.

45. Wu H, Xia T, Wei X, Chen L, Mo L, Yu B, et al. Relationship between diffusion-weighted imaging and $\mathrm{BCl}-2$ expression in rabbit models of hepatic VX2 tumors after radiotherapy. J Biol Regul Homeost Agents. 2013;27:487-95.

Ready to submit your research? Choose BMC and benefit from

- fast, convenient online submission

- thorough peer review by experienced researchers in your field

- rapid publication on acceptance

- support for research data, including large and complex data types

- gold Open Access which fosters wider collaboration and increased citations

- maximum visibility for your research: over 100M website views per year

At BMC, research is always in progress.

Learn more biomedcentral.com/submissions 